\title{
IMPUESTO A LA SALIDA DE DIVISAS E INVERSIÓN EXTRANJERA DIRECTA EN EL ECUADOR
}

\author{
TAX ON FOREIGN EXCHANGE AND FOREIGN DIRECT INVESTMENT IN ECUADOR
}

Lenin Estalin Fuentes Gavilanez ${ }_{2}^{1}$

Cristian Hugo Morales Alarcón ${ }^{2}$

\section{Resumen}

Se analiza el efecto del impuesto a la salida de divisas (ISD) sobre el nivel de inversión extranjera directa (IED) en el Ecuador, durante el período comprendido de 2008 a 2017. Para el efecto se realiza un estudio correlacional aplicando un modelo de regresión con las variables de impuesto a la salida de divisas en el Ecuador, y la inversión extranjera directa. Se puede determinar que el comportamiento de la inversión extranjera directa con respecto a los cambios en el impuesto a la salida de divisas durante el período de análisis no es significativo, lo cual explica que la variable independiente ISD no tiene incidencia dentro del modelo.

\section{Palabras clave}

Inversión Extranjera Directa, Impuesto a la Salida de Divisas, Análisis correlacional.

\section{Abstract}

The effect of the foreign currency exit tax (ISD) on the level of foreign direct investment (FDI) in Ecuador, during the period from 2008 to 2017, is analyzed. For this purpose, a correlational study is carried out, applying a regression model based on the variables of the tax on the outflow of foreign currency in Ecuador, with respect to the variations of foreign direct investment. It can be determined that the behavior of the foreign direct investment with respect to the changes in the tax on the exit of currencies during the period of analysis is not significant, which explains why the independent variable ISD has no incidence within the model.

\section{Keywords}

Foreign Direct Investment, Foreign Exchange Tax, Correlation Analysis.

\footnotetext{
${ }_{1}^{1}$ Universidad Nacional de Chimborazo (Ecuador). Correo electrónico: lenin.fuentes@unach.edu.ec

${ }^{2}$ Universidad Nacional de Chimborazo (Ecuador). Correo electrónico: cmorales@unach.edu.ec
} 


\section{Introducción}

A nivel mundial los gobiernos aplican impuestos con una perspectiva de justicia económica, con la finalidad de generar rentas, redistribución, representación y reformulación de precios. Lo hacen con el objetivo de incrementar o reducir una determinada variable económica, lo cual puede generar efectos positivos o negativos en el crecimiento económico de un país.

A partir de la Ley de Transformación Económica, expedida el 13 de marzo de 2000, se estableció un nuevo esquema de gestión macroeconómica que es necesario evaluar. Sin duda ésta ley impuso un cambio radical en la política económica del país (Acosta y Marconi, 2001). A partir del año 2000, la economía del Ecuador logró una ligera estabilización después de haber sufrido la peor crisis económica de su historia a finales de la década de los 90; una crisis que cambió radicalmente la vida de todos los ecuatorianos debido a que fue el detonante para que el país adoptase el dólar como moneda oficial, renunciando así a su soberanía monetaria.

La Constitución del Ecuador, expedida en el año 2008 luego de un proceso constituyente, determina cambios profundos en la concepción del modelo económico del Estado ecuatoriano, junto con otros como la organización estatal, el régimen de derechos civiles y otros, todos estos enfocados por una nueva noción de bienestar que gira alrededor de conceptos adecuados a una nueva visión de desarrollo, tales como la inclusión social, la equidad, la participación ciudadana, entre otras políticas sociales que buscan una distribución equitativa y promoción de las condiciones de vida de los habitantes (Bello, 2012).

El Producto Interno Bruto (PIB), es el principal indicador del comportamiento de la economía de un país, "es el indicador más efectivo para medir la capacidad de producción de bienes y servicios de un país. Es la suma de los valores monetarios del consumo, la inversión bruta, las compras de bienes y servicios por parte del Estado y las exportaciones netas producidas en un país durante un determinado año" (Samuelson y Nordhaus, 2001). Este permite evaluar el crecimiento que ha mantenido un país a lo largo del tiempo. Según los datos de evolución del PIB durante el período 2001 a 2017, obtenidos del Banco Central del Ecuador, existe una clara división de dos períodos después de la grave crisis económica que sufrió el país. La primera durante el período 2000 a 2006, dentro del cual los gobiernos de turno implementaban políticas neoliberales que contribuyeron a que el PIB del país registre un crecimiento promedio del 4.78\%; y el segundo, durante el período 2007 a 2017, que se caracteriza por la implementación de una reforma estructural de la economía, en parte en respuesta a la ideología del Gobierno de turno, presidido por el economista Rafael Correa Delgado quien, desde sus inicios, hizo una fuerte crítica al modelo neoliberal aplicado y propuso una economía de mercado con fuerte regulación estatal, donde este a su vez juega un papel activo como proveedor de infraestructura productiva.

En menos de 10 años, se pusieron en marcha alrededor de 22 reformas que cambiaron significativamente la dinámica operacional de los agentes económicos en el Ecuador, entre las cuales se pueden destacar la redistribución a través de impuestos, restricciones a la salida de divisas o capitales golondrina, el incentivo a nuevas inversiones, la lucha contra la evasión, el equilibrio fiscal o incentivos a los consumidores para que adquieran productos $y$ servicios hechos en Ecuador.

El impuesto a la salida de divisas (ISD) es un tributo que se aplica sobre el valor de las operaciones y transacciones monetarias que se realicen al exterior (Palma y Castro, 2016); es decir, el hecho generador de este impuesto lo constituye la transferencia, envío o traslado de divisas que se efectúen externamente del país, sea en efectivo o a través del giro de cheques, transferencias, retiros o pagos de cualquier naturaleza, inclusive compensaciones internacionales, sea que dicha operación se realice o no con la intervención de las instituciones que integran el sistema financiero.

La tarifa del impuesto a la salida de divisas fue aumentando progresivamente hasta alcanzar el 5\%. Este tributo debe ser pagado por todas las personas naturales; sucesiones indivisas; y sociedades privadas, nacionales y extranjeras, según lo dispuesto en el ordenamiento jurídico. Las instituciones financieras (IFI'S), así como el Banco Central, se constituyen en agentes de retención del impuesto cuando transfieran divisas al exterior por disposición de terceros. Las empresas de courier que envíen divisas al exterior por orden de sus clientes, se constituyen en agentes de percepción. Las personas que salgan del país llevando consigo más de tres salarios básicos unificados (USD 1.098), deben abonar este impuesto. Las personas naturales y las sociedades que contraten, promuevan o administren un espectáculo público, con la participación de personas no residentes en el Ecuador, también se constituyen en agentes de 
retención.

A su vez, el nivel de inversión extranjera directa (IED) es considerado como un componente esencial del motor del crecimiento de cualquier país en el mundo (Conferencia de las Naciones Unidas sobre Comercio y Desarrollo - UNCTAD, 2011). En América Latina la IED ha sido considerada como un factor indispensable para el desarrollo. De ella se ha esperado la modernización industrial, la importación de tecnología, la creación de empleos y se ha destacado su importante contribución al ahorro y al equilibrio de la balanza de pagos (Morales, 2010).

Ahora bien, para Villafuerte (2018), el impuesto a la salida de divisas es un tributo que incentiva el consumo y la inversión nacional. Afirma que es prioritario que la política tributaria sea estable; así los actores extranjeros y nacionales tienen una idea clara de las reglas del juego y pueden planificar sus estrategias empresariales. Por su parte Gutman, López \& Ramos (2016), señalan que, para el caso ecuatoriano, una medida para incentivar la inversión extranjera directa sería la reducción o exoneración del impuesto a la salida de divisas.

En este marco Giraldo y Obando (2012), diferencian la inversión extranjera directa vertical, la cual está dirigida a mercados que tienen acceso a una fuente importante de recursos naturales, de la horizontal, en la cual las motivaciones son muy diferentes pues el interés fundamental es acceder a mercados donde puedan vender sus productos. Concluyen que, tanto en la inversión extranjera vertical como en la horizontal, los inversionistas buscan países que tengan un sistema político estable, que les permita realizar inversiones a largo plazo. Para el caso de China, las regiones que han mejorado sus derechos de propiedad intelectual han recibido un mayor flujo de IED.

Empíricamente, Torres y Ochoa (2017), realizan un análisis correlacional de Pearson, a partir del cual comprueban la existencia de una relación negativa entre el IED y la Balanza Comercial. De igual modo, y mediante la estimación de un modelo de vectores autoregresivos con datos de panel, entre las variables de inversión extranjera directa, grado de apertura y crecimiento económicos, Cruz, Mendoza y González (2019), encuentran evidencia de una relación dinámica para la muestra total de países y para los países con nivel de crecimiento alto, pero no para aquellos con crecimiento bajo y medio. Encuentran, además, un efecto negativo de la IED sobre la inversión doméstica. Otros estudios similares realizados en Malasia (Sharma, Nayagam y Chung, 2012) y Europa central (Mateev, 2009) encuentran que la apertura comercial no tuvo impacto estadísticamente significativo sobre la IED en los países respectivos. Narudeen (2011), al estudiar los determinantes de la IED en Nigeria, muestran un fuerte vínculo entre el nivel de apertura comercial y las entradas de flujos de efectivo como parte de IED.

Parece existir un consenso general sobre la relevancia de la apertura comercial para atraer inversionistas extranjeros a los países anfitriones (Omán, 2000; Cohen, 2007; Dunning y Lundan, 2008). A medida que más cambios y políticas instituyan los gobiernos en favor de la apertura de sus economías, mayor será la probabilidad de recibir grandes sumas de IED en términos de cantidad y calidad.

Por otra parte, con respecto a las políticas fiscales vigentes en los países anfitriones, y como estas inciden en los flujos de inversión extranjera, existen varias estimaciones empíricas que muestran que las empresas generalmente buscan mercados con tasas de impuestos más bajas en comparación con su país de origen. Los gobiernos de los respectivos países anfitriones pretenden lograr un equilibrio entre las tasas de incentivos fiscales y la necesidad de recaudar una cantidad deseable de ingresos que sea asequible desde la perspectiva de las empresas multinacionales para proporcionar un entorno competitivo (UNCTAD, 2000). El informe de la UNCTAD (2000) y la investigación de Egger y Raff (2011) muestran una competencia muy alta entre los países en el marco del tratamiento del régimen de impuestos aplicados a las sociedades para alentar las entradas de IED en sus respectivos mercados.

En este contexto, la aplicación de un tributo en un país puede afectar de forma significativa, sea de manera positiva o negativa, al ingreso de IED a un país. Por ello, el objetivo de esta investigación es realizar un análisis del efecto que provocó el impuesto a la salida de divisas (ISD) sobre el nivel de inversión extranjera directa (IED) en el Ecuador, durante el período comprendido de 2008 a 2017.

\section{Metodología}

Se han seguido los siguientes pasos con el fin de determinar en qué medida el impuesto a la salida de divisas (ISD) 
ha incidido en la inversión extranjera directa (IED) en el Ecuador:

1. Revisión documental y construcción del marco teórico que guía la investigación respecto de la relación entre las variables en estudio: el impuesto a la salida de divisas como la inversión extranjera directa.

2. Búsqueda de información estadística de las variables y construcción de la base de datos. El impuesto a la salida de divisas se expresa en miles de dólares, y la inversión extranjera directa como porcentaje del PIB durante el periodo de estudio.

3. Determinación del coeficiente de correlación entre el impuesto a la salida de divisas y la inversión extranjera directa, mediante un proceso estadístico de regresión. La finalidad es estimar las relaciones entre las dos variables de estudio y determinar en qué medida el impuesto a la salida de divisas incide en la inversión extranjera directa en el Ecuador, durante el periodo comprendido desde el año 2008 al 2017.

En esta investigación se hará uso de un análisis descriptivo para identificar las tendencias claves en los datos estadísticos ya existentes del Banco Mundial, Banco Central del Ecuador y el Servicio de Rentas Internas. Se observará el comportamiento de los fenómenos estudiados y se obtendrá el coeficiente de correlación para determinar la medida de relación entre las dos variables en estudio. En este contexto se plantean las siguientes hipótesis estadísticas: Hipótesis nula (H0): No existe relación entre las variaciones del monto de recaudación del impuesto a la salida de divisas con el monto de inversión extranjera directa. Hipótesis alternativa (H1): Existe relación entre las variaciones del monto de recaudación del impuesto a la salida de divisas con el monto de la inversión extranjera directa.

\section{Resultados y discusión}

\section{Impuesto a la salida de divisas}

En el año 2008 entró en vigor el Impuesto a la Salida de Divisas (ISD), mecanismo establecido por el Gobierno de Rafael Correa con la finalidad de reducir la fuga de capitales al exterior y mantener liquidez interna. Al inicio, el ISD gravaba con un $0,5 \%$ a toda transferencia, envío o traslado de divisas que se efectúen hacia el exterior, ya sea en efectivo o a través del giro de cheques, retiros o pagos de cualquier naturaleza, inclusive a compensaciones internacionales. Esta herramienta permitió recaudar \$ 31.408,61 dólares. En 2009 subió al 1\%, con el cual se logró recaudar \$ 188.287,3 dólares. Un año más tarde pasó al 2\% y desde el 2012 es del 5\%, razón por la cual desde ese año la recaudación se incrementó en un 135\% comparado con el año 2011, alcanzando el \$1.159.590,5.

Figura 1. Impuesto a la salida de divisas (miles de dólares) - período 2008-2018.

ISD

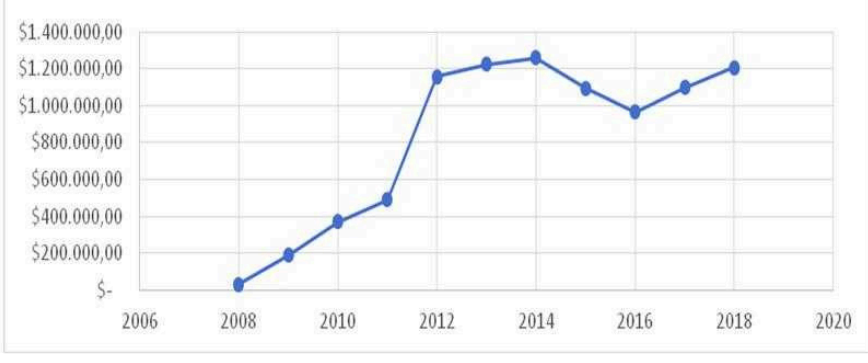

Fuente: Elaboración propia con base en el Servicio de Rentas Internas del Ecuador.

\section{Inversión extranjera directa en el Ecuador}

La División de las Naciones Unidas para el Comercio y el Desarrollo UNCTAD (2002), manifiesta que "la inversión extranjera directa refleja el interés a largo plazo de una entidad residente en una economía (inversor directo) en una entidad residente en otra economía (inversión directa). Cubre todas las transacciones entre los inversores directos y la inversión directa, lo cual significa que cubre no sólo la transacción inicial, sino que también las transacciones subsecuentes entre las dos entidades y el resto de las empresas afiliadas". En este apartado se describe y cuantifica los flujos netos de inversión extranjera directa en el Ecuador durante el período 20082018, para posteriormente evaluar si existe algún impacto de la reforma realizada al impuesto a la salida de divisas sobre los niveles de IED recibidos por el Ecuador durante el período de análisis.

Sin duda, América Latina y el Caribe han sido grandes beneficiarios de los flujos de inversión extranjera directa, lo cual ha permitido que la región cuente con una bonanza de capitales extranjeros. Lamentablemente existen fondos de capital de riesgo denominados "golondrina", mismos que realizan inversiones a corto plazo, y pretenden obtener rendimientos elevados, lógicamente a un mayor riesgo. Estos capitales por lo general crean una economía 
ficticia, generando inflación a través del mejoramiento de salarios con un efecto temporal ya que después de haber obtenido rentabilidad, los fondos de inversión se retiran rápidamente desestabilizando la economía interna de los países.

Tabla 1. Ingreso de inversión extranjera directa en América Latina durante el período 2008 a 2017, medido en millones de dólares.

\begin{tabular}{ccccccccccc}
\hline Pais & $\mathbf{2 0 0 8}$ & $\mathbf{2 0 0 9}$ & $\mathbf{2 0 1 0}$ & $\mathbf{2 0 1 1}$ & $\mathbf{2 0 1 2}$ & $\mathbf{2 0 1 3}$ & $\mathbf{2 0 1 4}$ & $\mathbf{2 0 1 5}$ & $\mathbf{2 0 1 6}$ & $\mathbf{2 0 1 7}$ \\
\hline Brasil & 50,716 & 31,418 & $\mathbf{8 8 , 4 5 2}$ & 101,158 & $\mathbf{8 6 , 6 0 7}$ & 69,181 & 96,895 & 75,075 & 77,794 & 70,685 \\
\hline Chile & 15,150 & 12,887 & 15,725 & 23,444 & 28,457 & 19,362 & 22,342 & 20,457 & 12,374 & 6,419 \\
\hline Colombia & 10,565 & 8,035 & 6,430 & 14,648 & 15,039 & 16,209 & 16,325 & 11,732 & 13,850 & 14,013 \\
\hline Argentina & 9,726 & 4,017 & 11,333 & 10,840 & 15,324 & 9,822 & 5,065 & 11,979 & 3,260 & 11,517 \\
\hline Perú & 6,924 & 6,431 & 8,455 & 7,665 & 11,918 & 9,298 & 7,885 & 7,817 & 6,863 & 6,769 \\
\hline Venezuela & 2,083 & $-1,137$ & 1,583 & 5,885 & 4,986 & 2,145 & 390.00 & 3,764 & 1,587 & -68 \\
\hline Uruguay & 2,142 & 1,603 & 2,191 & 2,690 & 2,569 & 3,040 & 2,385 & 1,369 & -384 & $-878,3$ \\
\hline Bolvia & 512 & 423 & 621 & 858 & 1,060 & 1,750 & 72 & 1,012 & 335,36 & 725,64 \\
\hline Paraguay & 260,9 & 49 & 344 & 447 & 745 & 74 & 459 & 315 & 451,74 & 507,01 \\
\hline Ecuador & 1,057 & 308,54 & 165,83 & 644,07 & 567,48 & 727,03 & 772,26 & $1,322,49$ & 767,41 & 618,43
\end{tabular}

Fuente: elaboración propia con base en el Banco Mundial.

El país con la mayor inversión extranjera directa durante el periodo de estudio es Brasil y el que menor inversión extranjera directa ha recibido es Ecuador.

\section{Efectos del impuesto a la salida de divisas (ISD) sobre inversión extranjera directa en el Ecuador.}

Para poder entender de mejor manera el efecto que ha tenido el Impuesto a la salida de divisas (ISD) sobre la Inversión extranjera directa (IED) en el Ecuador, analizaremos cual fue la variación de la IED como porcentaje del PIB desde el momento en el que se implementó el ISD. Como se puede observar en la figura 2, a finales del 2008 entró en vigencia el ISD, mismo que tuvo una connotación mínima ya que inició en un $1 \%$. Pese a que el ISD pasa del $1 \%$ al $2 \%$ en el año 2009 , la caída de la Inversión extranjera directa como porcentaje del PIB puede ser explicada por otros factores como la crisis financiera internacional, que desembocó en que la IED se redujera en un 70,91\% con respecto al año 2008.
Figura 2. Inversión Extranjera como porcentaje del PIB - período 20082017.

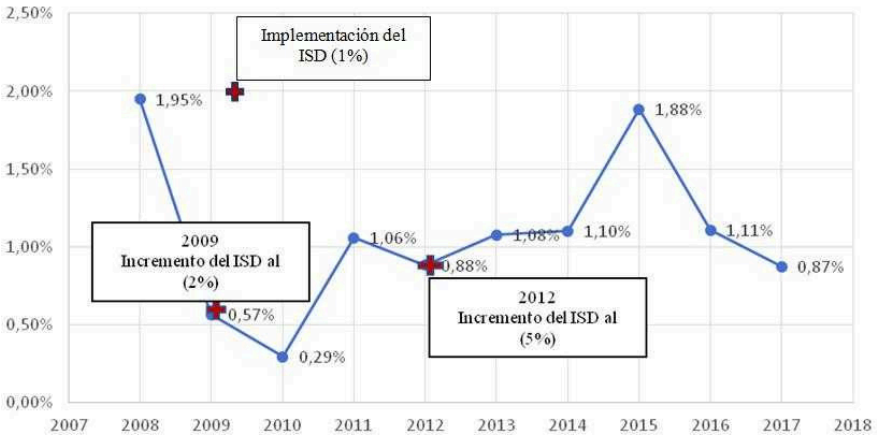

Fuente: elaboración propia con base en el Banco Mundial.

Para el año 2010, la crisis en cuanto a la atracción de capitales extranjeros al país se agudizó aún más debido a que el Gobierno de turno culminó los contratos con las compañías petroleras que, hasta entonces, operaban en el país (Petrobras, Canadá Grande y EDC).

En el año 2012, el ISD incrementa de nuevo y pasa del $2 \%$ al 5\%. Sin embargo, la IED en el año 2013, registra un ligero aumento con respecto al año anterior. En el año 2014 la IED vuelve a registrar una contracción debido a la inestabilidad de las políticas económicas implementadas por el régimen de turno. Para el año 2015, la IED vuelve a mantener un crecimiento sostenido debido a las grandes inversiones que se realizaron en los proyectos estratégicos del país.

A partir del año 2016, se puede observar una considerable disminución en el ingreso de capitales extranjeros al Ecuador. Esto en gran medida debido a la caída en los precios del petróleo a nivel internacional, el terremoto acontecido en abril del año en cuestión, y diversos contingentes legales que afectaron el desempeño de la actividad económica del país. Sectores como: agricultura, comercio, alojamiento, administración pública, construcción y comunicaciones fueron los más afectados.

Al suscitarse los acontecimientos mencionados con anterioridad, el índice riesgo país del Ecuador incrementó, razón por la cual los inversionistas extranjeros requerían un mayor Equity Market Risk Premium por las inversiones realizadas en el país. Esto contribuyó a que los flujos de IED se dirigieran hacia las naciones vecinas, donde podían obtener un mejor rendimiento del capital a un menor riesgo. 


\section{Análisis Estadístico del Impuesto a la salida de divisas sobre la Inversión extranjera directa en el Ecuador}

Para demostrar de una manera más precisa la relación que ha mantenido el Impuesto a la salida de divisas sobre la inversión extranjera directa en el Ecuador, a continuación se presenta un análisis estadístico de regresión, mismo que se efectúa a partir del 2008, año en que empieza la recaudación del ISD. El modelo de estimación empírico, que se construyó a partir de los datos obtenidos, parte del supuesto de que el impuesto a la salida de divisas y la inversión extranjera directa tienen una relación lineal, y que entre las dos variables existe una relación funcional que se puede representar de la siguiente manera:

$$
I E D=f(I S D)
$$

Tabla 2. Relación entre Inversión extrajera directa e impuesto de salida de divisas. Ecuador 2008 - 2017.

\begin{tabular}{ccccccc}
\hline $\begin{array}{c}\text { Regresión } \\
\text { mútiple } \\
\text { de IED } \\
\text { Resumen }\end{array}$ & $\begin{array}{c}\text { Múltiple } \\
\text { Res-Cuadrado }\end{array}$ & $\begin{array}{c}\text { R-cuadrado } \\
\text { ajustado }\end{array}$ & $\begin{array}{c}\text { Error } \\
\text { estadistico } \\
\text { de } \\
\text { estimación }\end{array}$ & $\begin{array}{c}\text { Filas } \\
\text { ignoradas }\end{array}$ & Marginales \\
\hline $\begin{array}{c}\text { Tabla } \\
\text { ANOVA }\end{array}$ & $\begin{array}{c}\text { Grados de } \\
\text { libertad }\end{array}$ & $\begin{array}{c}\text { Suma de } \\
\text { cuadrados }\end{array}$ & $\begin{array}{c}\text { Media de } \\
\text { cuadrados }\end{array}$ & $\mathbf{F}$ & Valor $\mathbf{p}$ & \\
\hline Explicado & 1 & $5,81843 \mathrm{E}+15$ & $5,81843 \mathrm{E}+15$ & 8,942 & 0,003 & \\
\hline $\begin{array}{c}\text { Tabla de } \\
\text { regresión }\end{array}$ & Coeficiente & $\begin{array}{c}\text { Error } \\
\text { estándar }\end{array}$ & Valor $\mathbf{t}$ & Valor $\mathbf{p}$ & Inferior & Superior \\
\hline Constante & 45833873,99 & 4664935,331 & 9,825 & $5,21066 \mathrm{E}-17$ & 36596032,02 & 55071716 \\
\hline ISD & 184,119 & 61,569 & 2,990 & 0,003 & 62,191 & 306,04 \\
\hline
\end{tabular}

Fuente: Elaboración propia.

De acuerdo con el modelo utilizado, donde fueron analizadas 120 observaciones, se puede determinar que el comportamiento de la inversión extranjera directa con respecto a los cambios en el impuesto a la salida de divisas durante el período de análisis no es significativo. Según la estimación realizada, el coeficiente de determinación $\mathrm{R}^{2}$ obtiene un valor de $0,7 \%$ lo cual explica que la variable independiente (ISD) no tiene incidencia dentro del modelo.

Tabla 3. Análisis de correlación y co-varianza entre la Inversión extranjera directa y el impuesto a la salida de divisas, Ecuador 2008 2017.

\begin{tabular}{ccc}
\hline $\begin{array}{c}\text { Tabla de correlación } \\
\text { lineal }\end{array}$ & $\begin{array}{c}\text { Inversión extranjera } \\
\text { directa (IED) }\end{array}$ & $\begin{array}{c}\text { Impuesto a la salida de } \\
\text { divisas (ISD) }\end{array}$ \\
\hline $\begin{array}{c}\text { Inversión extranjera } \\
\text { directa (IED) }\end{array}$ & 1,000 & 0,265416 \\
\hline $\begin{array}{c}\text { Impuesto a la salida de } \\
\text { divisas (ISD) }\end{array}$ & 0,265416 & 1,000 \\
\hline
\end{tabular}

El coeficiente de correlación toma valores entre -1 y 1 , por lo tanto, un valor de 1 indica una relación lineal perfectamente positiva, mientras que el valor de -1 indica una relación perfectamente negativa. Según la estimación realizada se puede observar en la tabla 3 , que el coeficiente de correlación entre la inversión extranjera directa y el impuesto a la salida de divisas es un número positivo cercano a cero $(0,2654)$, lo cual indica que la relación lineal es casi nula.

\section{Conclusiones}

De acuerdo con los resultados obtenidos de la regresión entre la inversión extranjera directa (IED) y el impuesto a la salida de divisas (ISD), a pesar de las fluctuaciones que registran ambas variables, se puede determinar que con una tasa del $5 \%$ en el impuesto a la salida de divisas, la inversión extranjera no se ve influenciada. No se podría asegurar el efecto que tendría en la inversión extranjera si el ISD vuelve a incrementarse y, por ejemplo, pasa del 5\% al $10 \%, 15 \%$ o $20 \%$.

Finalmente, después de haber realizado el análisis correspondiente con base en otros estudios, se puede concluir que la disminución de la IED en el Ecuador está ligada no solo al establecimiento de impuestos como el ISD, sino puede deberse a otros factores tales como: el estado del contrato social, el nivel intelectual del talento humano, el acceso a los consumidores, un ambiente político y social estable, la facilidad para hacer negocios, la calidad y confiabilidad de la infraestructura, los servicios básicos y el nivel de corrupción, los cuales en trabajos futuros serán analizados.

\section{Referencias}

1. Acosta, A., y Marconi Romano, S. (2001). Macroeconomía y economía política en dolarización. Abya-Yala/UPS.

2. Anderson, D. E. (1995). Extensible programming: Beyond reusable objects. Behavior Research Methods. Instruments, \& Computers, 27(2), 131-133.

3. Asamblea constituyente. (2008). Constitución de la República del Ecuador.

4. Bello, G. G. (2012). Las disposiciones legales que desarrollan los preceptos constitucionales sobre la inversión privada extranjera en el Ecuador. Foro 
Revista de Derecho, 17, 31-61.

5. Cruz Vásquez, M., Mendoza Velázquez, A., \& Pico González, B. (2019). Inversión extranjera directa, apertura económica y crecimiento económico en América Latina. Contaduría y administración, 64(1), 1-21.

6. Fundación, B. I. (2009). El ciclo inversor de la economía española, 1994-2008. Fundación BBVA.

7. Giraldo, D. E. J., \& Obando, H. R. (2012). Determinantes y efectos de la inversión extranjera directa: revisión de literatura. Ensayos de economía, 22(41), 109-128.

8. Gutman, V., López, A., \& Ramos, D. (2016). Análisis de los mecanismos de regulación y promoción de la Inversión Extranjera Directa (IED) en Ecuador. Inter-American Development Bank.

9. Interno Bruto Samuelson, P., \& Nordhaus, W. (2001). Macroeconomía. Madrid: McGRAW-Hill/ Interamericana de España.S.A

10. Morales, J. (2010). Inversión extranjera directa y desarrollo en América Latina. Problemas del Desarrollo, 41(163), 141-156.

11. Narudeen, A., Wafure, O. G., \& Auta, E. M. (2011). Determinants of Foreign Direct Investment: The Case of Nigeria. IUP Journal of Monetary Economics, 9(3), 50-67.

12. Palisade. (2019). StatTools: Forecasting and Statistical Analysis Software for Excel - Palisade.

13. Palma, H., \& Castro, F. (2016). Impacto Económico del Impuesto a la Salida de Divisas. Trabajo de titulación especial para obtención del grado de magíster en tributación y finanzas. Universidad de Guayaquil.

14. Pérez, R., Caso, C., Río, M. J., \& López, A. J. (2011). Introducción a la estadística económica. Dpto. de Economía Aplicada, Universidad de Oviedo.

15. Phillip Copeland RAYMOND MARBURY, by E., \& Mentor, F. (2016). An Investigation About the Small Business Adoption of Mobile Commerce.

16. Sahoo, P. (2006). Foreign Direct Investment in South Asia: Policy, Trends, Impact and Determinants (N.56). ADB Institute Discussion Papers.

17. Torres Ontaneda, W. I.; Ochoa Moreno, W. S. (2017). Efectos del impuesto a la salida de divisas en la balanza comercial intersectorial. Yura: Relaciones Internacionales, 11, 377-397

18. UNCTAD - Conferencia de las Naciones Unidas sobre Comercio y Desarrollo (2011) Informe sobre las inversiones en el mundo 2011. Panorama general. Formas no accionarias de producción internacional y Desarrollo. Nueva York y Ginebra: Naciones Unidas.

19. UNCTAD - Conferencia de las Naciones Unidas sobre Comercio y Desarrollo (2017). World Investment Report: Investment in Digital Economy. New York and Geneva: United Nations.

20. Villafuerte, D. E. (2018). El impuesto a la salida de divisas, entre la regulación extrafiscal y la recaudación. Revista San Gregorio, (26), 60-67. 